\title{
Oscillation Test for Linear Delay Differential Equation with Nonmonotone Argument
}

\author{
NURTEN KILIÇ (1) \\ Department of Mathematics, Faculty of Art and Science, Kutahya Dumlupinar University, 43100, Kutahya, Turkey.
}

Received: 08-03-2021 • Accepted: 18-08-2021

Aвstract. In this article, we analyze the first order linear delay differential equation

$$
x^{\prime}(t)+p(t) x(\tau(t))=0, t \geq t_{0},
$$

where $p, \tau \in C\left(\left[t_{0}, \infty\right), \mathbb{R}^{+}\right)$and $\tau(t) \leq t, \lim _{t \rightarrow \infty} \tau(t)=\infty$. Under the assumption that $\tau(t)$ is not necessarily monotone, we obtain new sufficient criterion for the oscillatory solutions of this equation. We also give an example illustrating the result.

2010 AMS Classification: $34 \mathrm{~K} 06,34 \mathrm{~K} 11$

Keywords: Delay equation, nonmonotone argument, oscillatory solution, nonoscillatory solution.

\section{INTRODUCTION}

The theory of oscillation is an important research area for applied mathematics. Also, substantial concern has been dedicated to the oscillatory and nonoscillatory solutions of some classes of differential equations. Particularly, delay differential equations have attracted a lot of scientists in recent years. Delay differential equations are differential equations where derivative functions rely on not only present value, but also on the previous value. See, for example [1-17], and the references cited therein. The reader is referred to monograph [8] for the general information about oscillation theory.

Consider the linear delay differential equation

$$
x^{\prime}(t)+p(t) x(\tau(t))=0, t \geq t_{0}
$$

where the functions $p, \tau \in C\left(\left[t_{0}, \infty\right), \mathbb{R}^{+}\right)$and $\tau(t)$ is not necessarily monotone such that

$$
\tau(t) \leq t \text { for } t \geq t_{0}, \lim _{t \rightarrow \infty} \tau(t)=\infty .
$$

By a solution of (1.1), we mean continuously differentiable function defined on $\left[\tau\left(T_{0}\right), \infty\right)$ for some $T_{0} \geq t_{0}$ such that (1.1) holds for $t \geq T_{0}$. A solution of (1.1) is called oscillatory if it has arbitrarily large zeros. Otherwise, it is called nonoscillatory.

The first systematic study for the oscillation of all solutions of (1.1) was made by Myshkis in 1950. Later, Koplatadze and Chanturija [11], Fukagai and Kusano [7], Ladde et al. [14] and Györi and Ladas [8] analyzed this equation and 
obtained some well-known oscillation criteria when the delay argument is nondecreasing.

Let $\alpha$ and $\beta$ be defined by

$$
\alpha:=\liminf _{t \rightarrow \infty} \int_{\tau(t)}^{t} p(s) d s
$$

and

$$
\beta:=\limsup _{t \rightarrow \infty} \int_{\tau(t)}^{t} p(s) d s .
$$

In 1988, Erbe and Zhang [5] established the following condition.

If $0<\alpha \leq \frac{1}{e}$ and $\tau(t)$ is nondecreasing,

$$
\beta>1-\frac{\alpha^{2}}{4}
$$

then all solutions of (1.1) are oscillatory.

Since then, many authors have tried to obtain better results by improving the upper bound for $\frac{x(\tau(t))}{x(t)}$. Also, in 1991, Chao [3] obtained the following condition

$$
\beta>1-\frac{\alpha^{2}}{2(1-\alpha)} .
$$

In 1992, Yu and Wang [16] and Yu et al. [17] found out the following one.

If $0<\alpha \leq \frac{1}{e}$ and $\tau(t)$ is nondecreasing,

$$
\beta>1-\frac{1-\alpha-\sqrt{1-2 \alpha-\alpha^{2}}}{2},
$$

then all solutions of (1.1) are oscillatory.

In 1990, Elbert and Stavroulakis [4] and in 1991, Kwong [13] established the following criteria by using different techniques, respectively. When $0<\alpha \leq \frac{1}{e}$ and $\tau(t)$ is nondecreasing

$$
\beta>1-\left(1-\frac{1}{\sqrt{\lambda_{1}}}\right)^{2}
$$

and

$$
\beta>\frac{\ln \lambda_{1}+1}{\lambda_{1}},
$$

where $\lambda_{1}$ is the smaller root of equation $\lambda=e^{\alpha \lambda}$.

In 1994 Koplatadze and Kvinikadze [12] improved (1.2). Moreover, in 1998 Philos and Sficas [15], in 1999, Jaroš and Stavroulakis [9] and in Kon et al. [10] obtained the following conditions for oscillatory solutions of (1.1) when $0<\alpha \leq \frac{1}{e}$ and $\tau(t)$ is nondecreasing.

$$
\begin{gathered}
\beta>1-\frac{\alpha^{2}}{2(1-\alpha)}-\frac{\alpha^{2}}{2} \lambda_{1}, \\
\beta>\frac{\ln \lambda_{1}+1}{\lambda_{1}}-\frac{1-\alpha-\sqrt{1-2 \alpha-\alpha^{2}}}{2},
\end{gathered}
$$

and

$$
\beta>2 \alpha+\frac{2}{\lambda_{1}}-1,
$$

where $\lambda_{1}$ is the smaller root of equation $\lambda=e^{\alpha \lambda}$.

When the delay argument $\tau(t)$ is not necessarily monotone, the result which was presented by Chatzarakis and Péics [2] includes (1.3).

Thus, in this paper our aim is to essentially develop these results under the assumption that $\tau(t)$ is not necessarily monotone argument. 


\section{Main Results}

In this section, we study the differential equation (1.1) with nonmonotone delay. Set

$$
h(t):=\sup _{s \leq t}\{\tau(s)\}, t \geq 0 .
$$

Clearly, $h(t)$ is nondecreasing and $\tau(t) \leq h(t)$ for all $t \geq 0$.

The following results will be useful to obtain main results.

Lemma 2.1. [6, Lemma 2.1.1]

$$
\liminf _{t \rightarrow \infty} \int_{\tau(t)}^{t} p(s) d s>0
$$

Then, we have

$$
\liminf _{t \rightarrow \infty} \int_{\tau(t)}^{t} p(s) d s=\liminf _{t \rightarrow \infty} \int_{h(t)}^{t} p(s) d s .
$$

Lemma 2.2. [1, Lemma 2] (See, also [13, Lemma 1]) Suppose that $\alpha>0$ and (1.1) has an eventually positive solution $x(t)$. Then, $\alpha \leq \frac{1}{e}$ and

$$
\liminf _{t \rightarrow \infty} \frac{x(h(t))}{x(t)} \geq \lambda_{1}
$$

where $\lambda_{1}$ is the smaller root of equation $\lambda=e^{\alpha \lambda}$.

Lemma 2.3. Let $0<\alpha \leq \frac{1}{e}$ and $x(t)$ be an eventually positive solution of (1.1). Assume that there exists $\theta>0$ such that

$$
\int_{h(u)}^{h(t)} p(s) d s \geq \theta \int_{u}^{t} p(s) d s \text { for all } h(t) \leq u \leq t .
$$

Then,

where $K$ is given by

$$
\limsup _{t \rightarrow \infty} \frac{x(h(t))}{x(t)} \leq \frac{2}{1-\alpha-\sqrt{(1-\alpha)^{2}-4 K}}
$$

$$
K=\frac{e^{\lambda_{1} \theta \alpha}-\lambda_{1} \theta \alpha-1}{\left(\lambda_{1} \theta\right)^{2}}
$$

and $h(t)$ is defined by (2.1).

Proof. Let $\delta: 0<\delta<\alpha$ be any number of arbitrarily close to $\alpha$ and $T>t_{0}$ large enough so that $h(t)>t_{0}$ and also from Lemma 2.1, we have $\int_{h(t)}^{t} p(s) d s>\delta$ for every $t \geq T$. Let $t \geq T$ and $T_{1} \equiv T_{1}(t)>t: h\left(T_{1}\right)=t$. Since $\int_{t}^{T_{1}} p(s) d s>\delta$, there exists $T_{1}>t_{1} \equiv t_{1}(t)>t$ such that

$$
\int_{t}^{t_{1}} p(s) d s=\delta
$$

Since $h(t) \geq \tau(t)$ and $x(t)$ is nonincreasing, from (1.1), we have

$$
x^{\prime}(t)+p(t) x(h(t)) \leq 0 .
$$

Integrating (2.4) from $t$ to $t_{1}$, we obtain

$$
x\left(t_{1}\right)-x(t)+\int_{t}^{t_{1}} p(s) x(h(s)) d s \leq 0
$$


or

$$
x(t) \geq x\left(t_{1}\right)+\int_{t}^{t_{1}} p(s) x(h(s)) d s .
$$

Also, integrating (2.4) from $h(s)$ to $t$ for $s<t_{1}$, we get

$$
x(t)-x(h(s))+\int_{h(s)}^{t} p(u) x(h(u)) d u \leq 0
$$

or

$$
x(h(s)) \geq x(t)+\int_{h(s)}^{t} p(u) x(h(u)) d u
$$

Combining (2.5) and (2.6), we have

$$
x(t) \geq x\left(t_{1}\right)+\int_{t}^{t_{1}} p(s)\left(x(t)+\int_{h(s)}^{t} p(u) x(h(u)) d u\right) d s .
$$

Let $0<\lambda<\lambda_{1}$. Then, the function

By Lemma 2.2

$$
\varphi(t)=x(t) e^{\lambda \int^{t} p(s) d s}, t \geq a
$$

$$
\frac{x(h(t))}{x(t)}>\lambda
$$

for all sufficiently large $t$ and then

$$
0=x^{\prime}(t)+p(t) x(\tau(t)) \geq x^{\prime}(t)+p(t) x(h(t))>x^{\prime}(t)+\lambda p(t) x(t)
$$

and also

$$
\varphi^{\prime}(t)=e^{\lambda \int_{t_{0}}^{t} p(s) d s}\left(x^{\prime}(t)+\left.x(t) \lambda p(s)\right|_{t_{0}} ^{t}\right) \leq 0
$$

which implies $\varphi^{\prime}(t) \leq 0$ for all sufficiently large $t$, that is $\varphi(t)$ is nonincreasing.

Since

$$
\varphi(h(t))=x(h(t)) e^{\lambda \int_{t_{0}}^{h(t)} p(s) d s} \Rightarrow x(h(t))=\varphi(h(t)) e^{-\lambda \int_{t_{0}}^{h(t)} p(s) d s},
$$

by using (2.3) and (2.8) in (2.7), we obtain

$$
x(t) \geq x\left(t_{1}\right)+\delta x(t)+\varphi(h(t)) \int_{t}^{t_{1}} p(s)\left(\int_{h(s)}^{t} p(u) e^{-\lambda \int_{t_{0}}^{h(u)} p(\xi) d \xi} d u\right) d s .
$$

Also, we know that

$$
e^{-\lambda \int_{t_{0}}^{h(u)} p(\xi) d \xi}=e^{-\lambda \int_{t_{0}}^{h(t)} p(\xi) d \xi+\lambda \int_{h(u)}^{h(t)} p(\xi) d \xi}
$$

By using this fact in (2.9), we have

$$
x(t) \geq x\left(t_{1}\right)+\delta x(t)+\varphi(h(t)) e^{-\lambda \int_{t_{0}}^{h(t)} p(s) d s} \int_{t}^{t_{1}} p(s)\left(\int_{h(s)}^{t} p(u) e^{\lambda \int_{h(t)}^{h(t)} p(\xi) d \xi} d u\right) d s
$$

and so

$$
x(t) \geq x\left(t_{1}\right)+\delta x(t)+x(h(t)) \int_{t}^{t_{1}} p(s)\left(\int_{h(s)}^{t} p(u) e^{\lambda \int_{h(u)}^{h(t)} p(\xi) d \xi} d u\right) d s
$$


From (2.2), we have

$$
\begin{aligned}
\int_{h(s)}^{t} p(u) e^{\lambda \int_{h(u)}^{h(t)} p(\xi) d \xi} d u & \geq \int_{h(s)}^{t} p(u) e^{\lambda \theta \int_{u}^{t} p(\xi) d \xi} d u \\
& =\frac{1}{\lambda \theta}\left[e^{\lambda \theta \int_{h(s)}^{t} p(\xi) d \xi}-1\right]
\end{aligned}
$$

Then, since $\int_{h(t)}^{t} p(s) d s>\delta$ and (2.3), we have

$$
\begin{aligned}
\int_{t}^{t_{1}} p(s)\left(\int_{h(s)}^{t} p(u) e^{\lambda \int_{h(u)}^{h(t)} p(\xi) d \xi} d u\right) d s & \geq \frac{1}{\lambda \theta} \int_{t}^{t_{1}} p(s)\left[e^{\lambda \theta \int_{h(s)}^{t} p(\xi) d \xi}-1\right] d s \\
& =\frac{1}{\lambda \theta} \int_{t}^{t_{1}} p(s) e^{\lambda \theta \int_{h(s)}^{t} p(\xi) d \xi} d s-\frac{1}{\lambda \theta} \int_{t}^{t_{1}} p(s) d s \\
& =\frac{1}{\lambda \theta} \int_{t}^{t_{1}} p(s) e^{\lambda \theta \int_{h(s)}^{t} p(\xi) d \xi} d s-\frac{\delta}{\lambda \theta} \\
& =\frac{1}{\lambda \theta} \int_{t}^{t_{1}} p(s) e^{\lambda \theta \int_{h(s)}^{s} p(\xi) d \xi-\lambda \theta \int_{t}^{s} p(\xi) d \xi} d s-\frac{\delta}{\lambda \theta} \\
& \geq \frac{1}{\lambda \theta} e^{\lambda \theta \delta} \int_{t}^{t_{1}} p(s) e^{-\lambda \theta \int_{t}^{s} p(\xi) d \xi} d s-\frac{\delta}{\lambda \theta} \\
& =\frac{1}{\lambda \theta} e^{\lambda \theta \delta} \frac{1}{\lambda \theta}\left[1-e^{-\lambda \theta \int_{t}^{t_{1}} p(\xi) d \xi}\right]-\frac{\delta}{\lambda \theta} \\
& =\frac{e^{\lambda \theta \delta}}{(\lambda \theta)^{2}}\left[1-e^{-\lambda \theta \delta}\right]-\frac{\delta}{\lambda \theta} \\
& \frac{1}{\lambda \theta)^{2}} \\
& \\
& \\
& \\
& \\
&
\end{aligned}
$$

and from (2.10) we have

$$
x(t) \geq x\left(t_{1}\right)+\delta x(t)+x(h(t)) K^{*},
$$

where

From (2.11), we get

$$
K^{*}=\frac{e^{\lambda \theta \delta}-\lambda \theta \delta-1}{(\lambda \theta)^{2}}
$$

$$
(1-\delta) x(t) \geq K^{*} x(h(t))
$$

or

$$
\frac{x(t)}{x(h(t))} \geq \frac{K^{*}}{(1-\delta)}:=d_{1} .
$$

Since $h\left(t_{1}\right) \leq t \leq t_{1}$ and $x(t)$ is nonincreasing, $x\left(h\left(t_{1}\right)\right) \geq x(t) \geq x\left(t_{1}\right)$, then we have

$$
x\left(t_{1}\right) \geq \frac{K^{*}}{(1-\delta)} x\left(h\left(t_{1}\right)\right)=d_{1} x\left(h\left(t_{1}\right)\right) \geq d_{1} x(t) .
$$

By using (2.12) in (2.11), we get

$$
x(t) \geq d_{1} x(t)+\delta x(t)+x(h(t)) K^{*}
$$


or

$$
x(t)\left(1-d_{1}-\delta\right) \geq x(h(t)) K^{*}
$$

and

$$
\frac{x(t)}{x(h(t))} \geq \frac{K^{*}}{\left(1-d_{1}-\delta\right)}:=d_{2}
$$

By following this process, we obtain

$$
\frac{x(t)}{x(h(t))} \geq \frac{K^{*}}{\left(1-d_{n}-\delta\right)}:=d_{n+1}, \quad n=1,2, \ldots .
$$

$1 \geq d_{n}>d_{n-1}$ for $n=2,3, \ldots$, which means that $d_{n}$ is increasing and it has a limit, $\lim _{t \rightarrow \infty} d_{n}=d$. Then, we have

$$
d^{2}-(1-\delta) d+K^{*}=0
$$

or

$$
d=\frac{1-\delta-\sqrt{(1-\delta)^{2}-4 K^{*}}}{2}
$$

For sufficiently large $t$,

$$
\frac{x(t)}{x(h(t))} \geq \frac{1-\delta-\sqrt{(1-\delta)^{2}-4 K^{*}}}{2}
$$

and since $0<\delta<\alpha$ is arbitrarily close to $\alpha$, by writing $\lambda \rightarrow \lambda_{1}$ in the last inequality, we obtain

$$
\limsup _{t \rightarrow \infty} \frac{x(h(t))}{x(t)} \leq \frac{2}{1-\alpha-\sqrt{(1-\alpha)^{2}-4 K}},
$$

where $K=\frac{e^{\lambda_{1} \theta \alpha}-\lambda_{1} \theta \alpha-1}{\left(\lambda_{1} \theta\right)^{2}}$, so the proof is completed.

Theorem 2.4. Let $0<\alpha \leq \frac{1}{e}$ and there exists $\theta>0$ such that

$$
\int_{h(u)}^{h(t)} p(s) d s \geq \theta \int_{u}^{t} p(s) d s \text { for all } h(t) \leq u \leq t .
$$

If

$$
\limsup _{t \rightarrow \infty} \int_{h(t)}^{t} p(s) d s>\frac{\ln \lambda_{1}+1}{\lambda_{1}}-\frac{1-\alpha-\sqrt{(1-\alpha)^{2}-4 K}}{2}
$$

then all soutions of (1.1) are oscillatory, where $\lambda_{1}$ is the smaller root of equation $\lambda=e^{\alpha \lambda}, K=\frac{e^{\lambda_{1} \theta \alpha}-\lambda_{1} \theta \alpha-1}{\left(\lambda_{1} \theta\right)^{2}}$ and $h(t)$ is defined by (2.1).

Proof. Assume for the sake of contradiction that $x(t)$ is an eventually positive solution of (1.1). If $x(t)$ is an eventually negative solution of (1.1), the proof of the theorem can be done similarly as below. Then, we know from [9, Theorem 1]

$$
\limsup _{t \rightarrow \infty} \int_{h(t)}^{t} p(s) d s \leq \frac{\ln \lambda_{1}+1}{\lambda_{1}}-\liminf _{t \rightarrow \infty} \frac{x(t)}{x(h(t))} .
$$

Also, we know from Lemma 2.3

$$
\liminf _{t \rightarrow \infty} \frac{x(t)}{x(h(t))} \geq \frac{1-\alpha-\sqrt{(1-\alpha)^{2}-4 K}}{2} .
$$

So, using this fact, we observe that (2.14) contradicts to (2.13). Then, the proof is completed. 
Remark 2.5. If we take $\theta=1$, then

and so, (2.13) reduces to

$$
K=\frac{\lambda_{1}-\lambda_{1} \alpha-1}{\lambda_{1}^{2}}
$$

$$
\limsup _{t \rightarrow \infty} \int_{h(t)}^{t} p(s) d s>2 \alpha+\frac{2}{\lambda_{1}}-1 .
$$

Example 2.6. Consider linear delay differential equation

$$
x^{\prime}(t)+0.5 x\left(t-\cos ^{2} t-0.7\right)=0, t \geq 0
$$

Since

$$
\liminf _{t \rightarrow \infty} \int_{\tau(t)}^{t} p(s) d s=\liminf _{t \rightarrow \infty} 0.5\left(\cos ^{2} t+0.7\right)=0.35<\frac{1}{e}
$$

and

$$
\limsup _{t \rightarrow \infty} \int_{h(t)}^{t} p(s) d s=\limsup _{t \rightarrow \infty} 0.5\left(\cos ^{2} t+0.7\right)=0.85<1,
$$

then well-known oscillation criteria do not hold. Also, from $\lambda=e^{0.35 \lambda}$ we have $\lambda=2.04754$.

Hence, by using Remark 2.5 , we observe that

$$
\limsup _{t \rightarrow \infty} \int_{h(t)}^{t} p(s) d s=0.85>2 \alpha+\frac{2}{\lambda_{1}}-1 \approx 0.67678
$$

then all solutions of (2.15) are oscillatory.

\section{CONFLICTS OF INTEREST}

The author declares that there are no conflicts of interest regarding the publication of this article.

\section{Authors Contribution Statement}

All authors jointly worked on the results and they have read and agreed to the published version of the manuscript.

\section{REFERENCES}

[1] Akca, H., Chatzarakis, G.E., Stavroulakis, I.P., An oscillation criterion for delay differential equations with several non-monotone arguments, Applied Mathematics Letters, 59(2016), 101-108.

[2] Chatzarakis, G.E., Péics, H., Differential equations with several non-monotone arguments: An oscillation result, Applied Mathematics Letters, 68(2017), 20-26.

[3] Chao, J., On the oscillation of linear differential equations with deviating arguments, Math. in Practice and Theory, 1(1991), 32-40.

[4] Elbert, A., Stavroulakis, I.P., Oscillations of first order differential equations with deviating arguments, University of Ioannina, T.R. No 172 1990, Recent trends in differential equations, 163-178, World Sci. Ser. Appl. Anal., 1, World Sci. Publishing Co., 1992.

[5] Erbe, L.H., Zhang, B.G., Oscillation of first order linear differential equations with deviating arguments, Differ. Integral Equ., 1(1988), 305314.

[6] Erbe, L.H., Kong, Q., Zhang, B.G., Oscillation Theory for Functional Differential Equations, Marcel Dekker, New York, 1995.

[7] Fukagai, N., Kusano, T., Oscillation theory of first order functional differential equations with deviating arguments, Ann. Mat. Pura Appl., 136(1984), 95-117.

[8] Győri, I., Ladas, G., Oscillation Theory of Delay Differential Equations with Applications, Clarendon Press, Oxford, 1991.

[9] Jaroš, J., Stavroulakis, I.P., Oscillation tests for delay equations, Rocky Mountain J. Math., 29(1999), 139-145.

[10] Kon, M., Sficas, Y.G., Stavroulakis, I.P., Oscillation criteria for delay equations, Proc. Amer. Math. Soc., 128(2000), $2989-2997$.

[11] Koplatadze, R.G., Chanturija, T.A., Oscillating and monotone solutions of first-order differential equations with deviating arguments, (Russian), Differentsial'nye Uravneniya, 8(1982), 1463-1465.

[12] Koplatadze, R., Kvinikadze, G., On the oscillation of solutions of first order delay diferential inequalities and equations, Georgian Mathematical Journal, 1(6)(1994), 675-685.

[13] Kwong, M.K., Oscillation of first-order delay equations, J. Math. Anal. Appl., 156(1991), 274-286.

[14] Ladde, G.S., Lakshmikantham, V., Zhang, B.G., Oscillation Theory of Differential Equations with Deviating Arguments, Monographs and Textbooks in Pure and Applied Mathematics, vol. 110, Marcel Dekker, Inc., New York, 1987. 
[15] Philos, Ch.G., Sficas, Y.G., An oscillation criterion for first order linear delay differential equations, Canad. Math. Bull. 41(1998), $207-213$.

[16] Yu, J.S., Wang, Z.C., Some further results on oscillation of neutral differential equations, Bull. Aust. Math. Soc., 46(1992), $149-157$.

[17] Yu, J.S., Wang, Z.C., Zhang, B.G., Qian, X.Z., Oscillations of differential equations with deviating arguments, PanAmerican Math. J., 2(1992), 59-78. 